In regard to the point made by Dr. Thompson about the visual acuity, he did not consider it in itself a reliable test at all. Aś we know, one may find normal vision in eyes showing marked fundus changes such as choked disk, etc. In making a diagnosis the entire eye symptom complex viz. anesthesia of cornea, contracted visual fields, enlarged pupil, negative psychic, sensory, and Piltz pupillary reflexes and the appearance of the disk described should all be considered and not merely the fundus alone.

\title{
A FAMILY TYPE OF COMBINED SCLEROSIS - ASSOCIATED. WITH GRAVE ANEMIA
}

\section{By Charles L. Dana, M.D.}

Dr. Dana had recently had under his care a patient in whom it might be said that pernicious anemia and combined sclerosis was a family disease. The patient was an unmarried woman, 58 years old, coming from old New England and Pennsylvania stock. She had lived a temperate and fairly healthy life, though subject rather easily to diarrheas, and having at times what Dr. Delafield termed "morning diarrhea." Very early in life she had a severe attack of dysentery, but had had no subsequent attack until eight years ago. She had other attacks seven, six, five and four years ago, but none since. Besides the dysentery, within the past eight years, she had had a distinct tendency to mild attacks of enterocolitis, and two years ago she was treated for a long time for this trouble, during which period she passed a great deal of undigested food and gas in her stools. Her physician detected an ulcer in the sigmoid flexure. She recovered from this, however and had been fairly well for nearly a year. Within the past six months she had noticed a peculiar numbness of the feet, with weakness and unsteadiness in walking, and a similar condition in the hands. When Dr. Dana first saw her, she had a decided pallor, with an almost yellowish tinge to the skin. She was rather thin, but not much more so than her ordinary habit. Subjectively, she felt fairly well excepting for the weakness and uncomfortable numbness of the extremities. She had an ataxic gait, and the Brauch-Romberg symptom. There was also some ataxia of the hands, so that she could not sew or do fine work with them, but she was able to write and dress herself. There was some general weakness of the extremities, but no distinct paralysis. The knee jerks were present, but there was no ankle clonus and no Babinski. While she complained much of the paresthesia, she had no actual cutaneous anesthesia. Examination of the patient's blood showed $3,200,000$ red cells; 8,800 white cells and 66 per cent. hemoglobin. She had a curious and persistent inflammatory condition of the gums, which kept her mouth rather sore. Cultures made of this region by Dr. F. M. Huntoon showed the ordinary microörganisms of the mouth, excepting that there were certain spirilla. An examination of the stools made by Dr. Christian A. Herter showed indications of excessive putrefaction, with an abundance of the bacillis aerogenes capsulatus. There was an excess of hydro-bilirubin and skatol, with absence of indol. A second examination of the blood, made three weeks later, showed a further decrease of the red cells to $2,780,000$, with a similar condition of corpuscles, excepting that there were no megaloblasts. The hemoglobin was reduced to $5^{8}$ per cent. 
In this case, Dr. Dana said, the patient presented a very clear-cut type of grave anemia, with combined sclerosis. As to the family history the patient stated that her mother had also suffered from "morning diarrhea," and had a tendency to weakness of the bowels. Her father died of apoplexy. One brother died at the age of 52 from a trouble similar to her own, namely, he had a great deal of diarrhea followed by sensory disturbances, anemia, and weakness of the extremities. One sister died in the same way, and one maternal grandmother had a similar trouble. The disease, apparently, ran through three generations. In the second generation it produced only a moderate disturbance. In the third generation, out of four children, three had been affected. The case might be classed as a family form of pernicious anemia, or a family form of combined sclerosis, or, more properly, it was a family form of enteric weakness which secondarily led first to an anemia, and then to a spinal cord disorder. There were some authorities, however, who still asserted that the enteric trouble was not a primary one, but was secondary.

Dr. Joseph Fraenkel said that in connection with the subject presented he recalled a case which he had for some time under his observation at the Montefiore Home. It concerned a young man of about 25 years, who, while in South America had contracted an obstinate form of enterocolitis, which resisted treatment for quite some time. At the time of his admission to the Montefiore Home, he presented undoubted evidences of an organic disease of the spinal cord which fitted easiest a conception of a combined degeneration-exaggerated reflexes and hypotonia were interpreted in this sense. During the acute recrudescences of his colitic trouble, which were observed at the Montefiore Home at intervals of a few months, the spinal cord symptoms showed considerable amelioration. At the moment the speaker did not recollect whether there were any blood examination made, and if so, what the findings were.

Dr Abrahamson said he could conclude the history of the case mentioned by Dr. Fraenkel by stating that the patient finally died of tuberculosis. Among the later spinal symptoms he exhibited was analgesia of the toes. He had seen the patient at the German Hospital, where the case was regarded as one of amebic dysentery.

Dr. B. Sachs said he had never observed any of the family type of these cases of combined sclerosis, but the supposition thrown out by Dr. Dana that the condition was of gastro-enteric origin was very interesting. The speaker recalled the case of an extremely hysterical woman who for many years invariably vomited shortly after each meal. About two years ago, after a trip to Europe in a vain search for health, she returned in a much worse condition than before so far as her general nutrition was concerned, and developed marked spinal symptoms, with radiating pains in the upper and lower extremities, with more or less flaccid atrophy, and some slight involvement of the bladder. Blood examinations were made by two different men, and they could not agree whether the case was one of pernicious anemia or not. It was undoubtedly a case of grave anemia. The patient became weaker and weaker and finally died without any change in her paralytic condition. The upper extremities became almost completely paralyzed, while the power of the lower extremities was much impaired, and there was great pain, particularly in the arms. The speaker said he had regarded the case as one of spinal origin, due primarily to grave anemia.

Dr. Smith Ely Jelliffe, in discussing the fact that certain spirillæ were 
found in the cultures taken from the inflamed gums in the case reported by Dr. Dana, called attention to the fact that general spirillosis had recently been regarded as a cause of pernicious anemia.

Dr. Jacoby referred to the possible relationship of these cases to those in which there was a complete absence of hydrochloric acid, and in which a pernicious anemia developed. In the absence of gastric juice and hydrochloric acid, any pathogenic organisms that might be present would have' a free field for action, and from that point of view we should attribute some importance to the presence of achylia gastrica in connection with grave anemia. This theory would perhaps tend to corroborate the gastrointestinal etiology of the disease.

Dr. Dana, in closing, said that pernicious anemia could be associated with extreme atony of the gastric wall and achylia gastrica, and also with a similar condition of the intestinal walls. In the latter condition, the food was not absorbed, and passed through almost unchanged. Combined sclerosis, the speaker said, had been observed without the presence of gastric symptoms, and the toxemia in this spinal disease was usually of intestinal rather than gastric origin. The pernicious anemia that occurred in association with the gastric cases was in his. experience less often associated with cord symptoms. Some authorities did not agree with Herter that skatol was a dominant factor in pernicious anemia.

\section{THE RELATION OF TRAUMA TO ORGANIC DISEASES OF THE SPINAL CORD. SPINAL CORD TUMORS}

\section{By Pearce Bailey, M.D.}

The author stated that the ultimate effects of injury to the spinal cord could be divided into two classes: a first class, in which the trauma was followed, sooner or later, by a chronic progressive degeneration of the spinal cord elements, identical in symptoms and course with the same degeneration when it occurred when no injury had been received. The classical and much discussed type of this class was tabes; other types of this class which seemed to challenge less acrimonious discussion were progressive muscular atrophy, multiple sclerosis, ataxic paraplegia, syringomyelia, and, in fact, all the many chronic degenerative diseases. Any of these diseases might be inaugurated by injury or made worse by it, but for none of them was an exclusive traumatic origin demonstrable. Opinions for or against the view might be justifiable, but when all was said and done they were nothing but opinions, and the wisest might be in error.

Of the group mentioned, locomotor ataxia was the disease in which a traumatic origin was most improbable, and the speaker said he saw no means by which such an origin could be proved for it. Multiple sclerosis and progressive muscular atrophy, on the other hand, in which the onset was less insidious and the early symptoms more unmistakable, and which lacked the great underlying cause of tabes, lent themselves more easily to the assumption of trauma as a point of departure. Syringomyelia, also, from its close analogies to hematomyelia, offered a still more profitable field of study from the point of view of trauma. This disease merged toward the second class of diseases he had in mind, namely, those in which an injury was followed, not by a generalized degeneration, but by focal disease. Of course, he did not refer to acute surgical injuries of the 